\title{
Some generalizations of Suzuki and Edelstein type theorems
}

\section{Ovidiu Popescu}

\section{*Correspondence:}

ovidiu.popescu@unitbv.ro

Department of Mathematics and

Computer Sciences, Transilvania

University, Iuliu Maniu, Brasov,

Romania

\author{
Abstract \\ We prove some generalizations of Suzuki's fixed point theorem and Edelstein's \\ theorem. \\ MSC: $54 \mathrm{H} 25$ \\ Keywords: Banach principle; contraction; Suzuki's theorem; Edelstein's theorem
}

\section{Introduction and preliminaries}

Let $(X, d)$ be a complete metric space and $T$ be a selfmap of $X$. Then $T$ is called a contraction if there exists $r \in[0,1)$ such that

$$
d(T x, T y) \leq r d(x, y)
$$

for all $x, y \in X$.

The following famous theorem is referred to as the Banach contraction principle.

Theorem 1 (Banach [1]) Let $(X, d)$ be a complete metric space, and let $T$ be a contraction on $X$. Then $T$ has a unique fixed point.

This theorem is a very forceful and simple, and it has become a classical tool in nonlinear analysis. It has many generalizations, see [2-19].

In 2008, Suzuki [20] introduced a new type of mapping and presented a generalization of the Banach contraction principle in which the completeness can also be characterized by the existence of a fixed point of these mappings.

Theorem 2 [20] Let $(X, d)$ be a complete metric space, and let $T$ be a mapping on $X$. Define a nonincreasing function $\theta$ from $[0,1)$ onto $(1 / 2,1]$ by

$$
\theta(r)= \begin{cases}1 & \text { if } 0 \leq r \leq(\sqrt{5}-1) / 2 \\ (1-r) / r^{2} & \text { if }(\sqrt{5}-1) / 2 \leq r \leq 1 / \sqrt{2} \\ 1 /(1+r) & \text { if } 1 / \sqrt{2} \leq r<1\end{cases}
$$

Assume that there exists $r \in[0,1)$ such that $\theta(r) d(x, T x) \leq d(x, y)$ implies $d(T x, T y) \leq$ $r d(x, y)$ for all $x, y \in X$. Then there exists a unique fixed point $z$ of $T$. Moreover, $\lim _{n} T^{n} x=z$ for all $x \in X$.

O2013 Popescu; licensee Springer. This is an Open Access article distributed under the terms of the Creative Commons Attribution License (http://creativecommons.org/licenses/by/2.0), which permits unrestricted use, distribution, and reproduction in any medium, provided the original work is properly cited. 
Its further outcomes by Altun and Erduran [21], Karapinar [22, 23], Kikkawa and Suzuki [24, 25], Moț and Petruşel [26], Dhompongsa and Yingtaweesittikul [27], Popescu [28, 29], Singh and Mishra [30-32] are important contributions to metric fixed point theory.

Popescu [28] introduced a new type of contractive operator and proved the following theorem.

Theorem 3 [28] Let $(X, d)$ be a complete metric space and $T: X \rightarrow X$ be a $(s, r)$-contractive single-valued operator:

$$
x, y \in X \quad \text { with } d(y, T x) \leq s d(y, x) \quad \text { implies } \quad d(T x, T y) \leq r M_{T}(x, y)
$$

where $r \in[0,1), s>r$ and

$$
M_{T}(x, y)=\max \left\{d(x, y), d(x, T x), d(y, T y), \frac{d(x, T y)+d(y, T x)}{2}\right\} .
$$

Then $T$ has a fixed point. Moreover, if $s \geq 1$, then $T$ has a unique fixed point.

As a direct consequence of Theorem 3, we obtain the following result.

Theorem 4 Let $(X, d)$ be a complete metric space, and let $T$ be a mapping on $X$. Assume that there exist $r \in[0,1)$ and $s>r$ such that

$$
d(y, T x) \leq s d(y, x) \quad \text { implies } \quad d(T x, T y) \leq r d(x, y)
$$

for all $x, y \in X$. Then there exists a fixed point $z$ of $T$. Further, if $s \geq 1$, then there exists a unique fixed point of $T$.

The following theorem is a well-known result in fixed point theory.

Theorem 5 (Edelstein [33]) Let $(X, d)$ be a compact metric space, and let $T$ be a mapping on $X$. Assume $d(T x, T y)<d(x, y)$ for all $x, y \in X$ with $x \neq y$. Then $T$ has a unique fixed point.

Inspired by Theorem 2, Suzuki [34] proved a generalization of Edelstein's fixed point theorem (see also [35-38]).

Theorem 6 [34] Let $(X, d)$ be a compact metric space, and let $T$ be a mapping on $X$. Assume that $(1 / 2) d(x, T x)<d(x, y)$ implies $d(T x, T y)<d(x, y)$ for all $x, y \in X$. Then $T$ has a unique fixed point.

In this paper, we prove generalizations of Theorem 2, Theorem 4, Theorem 5 and extend Theorem 6 . The direction of our extension is new, very simple and inspired by Theorem 3.

\section{Main results}

We start this section by proving the following theorem.

Theorem 7 Let $(X, d)$ be a complete metric space, and let $T$ be a mapping on $X$. Assume that there exist $r \in[0,1), a \in[0,1], b \in[0,1),(a+b) r^{2}+r \leq 1$ if $r \in[1 / 2,1 / \sqrt{2}), a+(a+b) r \leq$ 
1 if $r \in[1 / \sqrt{2}, 1)$ such that

$$
a d(x, T x)+b d(y, T x) \leq d(y, x) \quad \text { implies } \quad d(T x, T y) \leq r d(x, y)
$$

for all $x, y \in X$. Then there exists a unique fixed point $z$ of $T$. Moreover, $\lim _{n} T^{n} x=z$ for all $x \in X$.

Proof Since $a d(x, T x)+b d(T x, T x)=a d(x, T x) \leq d(T x, x)$ holds for every $x \in X$, by hypothesis, we get

$$
d\left(T x, T^{2} x\right) \leq r d(x, T x)
$$

for all $x \in X$. We now fix $u \in X$ and define a sequence $\left\{u_{n}\right\} \in X$ by $u_{n}=T^{n} u$. Then (3) yields $d\left(u_{n}, u_{n+1}\right) \leq r^{n} d(u, T u)$, so $\sum_{n=1}^{\infty} d\left(u_{n}, u_{n+1}\right)<\infty$. Hence $\left\{u_{n}\right\}$ is a Cauchy sequence. Since $X$ is complete, $\left\{u_{n}\right\}$ converges to some point $z \in X$. We next show that

$$
d(T x, z) \leq r d(x, z)
$$

for all $x \in X, x \neq z$. Since $\lim _{n} d\left(u_{n}, T u_{n}\right)=0, \lim _{n} d\left(x, T u_{n}\right)=\lim _{n} d\left(x, u_{n}\right)=d(x, z)$, there exists a positive integer $v$ such that $a d\left(u_{n}, T u_{n}\right)+b d\left(x, T u_{n}\right) \leq d\left(x, u_{n}\right)$ for all $n \geq v$. By hypothesis, we get $d\left(T u_{n}, T x\right) \leq r d\left(u_{n}, x\right)$. Letting $n$ tend to $\infty$, we obtain $d(z, T x) \leq r d(z, x)$. That is, we have shown (4).

Now we assume that $T^{j} z \neq z$ for every integer $j \geq 1$. Then (4) yields

$$
d\left(T^{j+1} z, z\right) \leq r^{j} d(T z, z)
$$

for every integer $j \geq 1$. We consider the following three cases:

(a) $0 \leq r<1 / 2$,

(b) $1 / 2 \leq r<1 / \sqrt{2}$,

(c) $1 / \sqrt{2} \leq r<1$.

In the case (a) we note that $2 r<1$. Then, by (3) and (5), we have

$$
d(z, T z) \leq d\left(z, T^{2} z\right)+d\left(T z, T^{2} z\right) \leq r d(z, T z)+r d(z, T z)=2 r d(z, T z)<d(z, T z) .
$$

This is a contradiction.

In the case (b), we note that $2 r^{2}<1$. If we assume $a d\left(T^{2} z, T^{3} z\right)+b d\left(z, T^{3} z\right)>d\left(z, T^{2} z\right)$, then we have, in view of (3) and (5),

$$
\begin{aligned}
d(z, T z) & \leq d\left(z, T^{2} z\right)+d\left(T z, T^{2} z\right) \\
& <a d\left(T^{2} z, T^{3} z\right)+b d\left(z, T^{3} z\right)+d\left(T z, T^{2} z\right) \\
& \leq a r^{2} d(z, T z)+b r^{2} d(z, T z)+r d(z, T z) \\
& =\left[(a+b) r^{2}+r\right] d(z, T z) \\
& \leq d(z, T z) .
\end{aligned}
$$


This is a contradiction. Hence $a d\left(T^{2} z, T^{3} z\right)+b d\left(z, T^{3} z\right) \leq d\left(z, T^{2} z\right)$. By hypothesis and (5), we have

$$
\begin{aligned}
d(z, T z) & \leq d\left(z, T^{3} z\right)+d\left(T z, T^{3} z\right) \\
& \leq r^{2} d(z, T z)+r d\left(z, T^{2} z\right) \\
& \leq r^{2} d(z, T z)+r^{2} d(z, T z) \\
& =2 r^{2} d(z, T z) \\
& <d(z, T z) .
\end{aligned}
$$

This is also a contradiction.

In the case (c), we assume there exists an integer $v \geq 1$ such that

$$
a d\left(u_{n}, u_{n+1}\right)+b d\left(z, u_{n+1}\right)>d\left(z, u_{n}\right)
$$

for all $n \geq v$. Then

$$
\begin{aligned}
d\left(z, u_{n}\right) & <a d\left(u_{n}, u_{n+1}\right)+b\left[a d\left(u_{n+1}, u_{n+2}\right)+b d\left(z, u_{n+2}\right)\right] \\
& \leq(a+a b r) d\left(u_{n}, u_{n+1}\right)+b^{2} d\left(z, u_{n+2}\right) \\
& <(a+a b r) d\left(u_{n}, u_{n+1}\right)+b^{2}\left[a d\left(u_{n+2}, u_{n+3}\right)+b d\left(z, u_{n+3}\right)\right] \\
& \leq\left(a+a b r+a b^{2} r^{2}\right) d\left(u_{n}, u_{n+1}\right)+b^{3} d\left(z, u_{n+3}\right) .
\end{aligned}
$$

Continuing this process, we get

$$
\begin{aligned}
d\left(z, u_{n}\right) & <\left(a+a b r+a b^{2} r^{2}+\cdots+a b^{p-1} r^{p-1}\right) d\left(u_{n}, u_{n+1}\right)+b^{p} d\left(z, u_{n+p}\right) \\
& \leq a \frac{1-(b r)^{p}}{1-b r} d\left(u_{n}, u_{n+1}\right)+b^{p} d\left(z, u_{n+p}\right)
\end{aligned}
$$

for all $n \geq v, p \geq 1$. Letting $p$ tend to $\infty$, we obtain

$$
d\left(z, u_{n}\right) \leq \frac{a}{1-b r} d\left(u_{n}, u_{n+1}\right)
$$

for all $n \geq v$. Thus,

$$
d\left(z, u_{n+1}\right) \leq \frac{a}{1-b r} d\left(u_{n+1}, u_{n+2}\right) \leq \frac{a r}{1-b r} d\left(u_{n}, u_{n+1}\right)
$$

for all $n \geq v$, so

$$
\begin{aligned}
d\left(u_{n}, u_{n+1}\right) & \leq d\left(z, u_{n}\right)+d\left(z, u_{n+1}\right) \\
& <\frac{a}{1-b r} d\left(u_{n}, u_{n+1}\right)+\frac{a r}{1-b r} d\left(u_{n}, u_{n+1}\right) \\
& =\frac{a+a r}{1-b r} d\left(u_{n}, u_{n+1}\right) \\
& \leq d\left(u_{n}, u_{n+1}\right)
\end{aligned}
$$


for all $n \geq v$. This is a contradiction. Hence there exists a subsequence $\left\{u_{n(k)}\right\}$ of $\left\{u_{n}\right\}$ such that

$$
\operatorname{ad}\left(u_{n(k)}, u_{n(k)+1}\right)+b d\left(z, u_{n(k)+1}\right) \leq d\left(z, u_{n(k)}\right)
$$

for all $k \geq 1$. By hypothesis, we get $d\left(T z, T u_{n(k)}\right) \leq r d\left(z, u_{n(k)}\right)$ for all $k \geq 1$. Letting $k$ tend to $\infty$, we get $d(z, T z)=0$, that is, $z=T z$. This is a contradiction.

Thus there exists an integer $j \geq 1$ such that $T^{j} z=z$. By (3) we get $d(z, T z)=d\left(T^{j} z, T^{j+1} z\right) \leq$ $r^{j} d(z, T z)$, so $d(z, T z)=0$, that is, $T z=z$.

Now we suppose that $y$ is another fixed point of $T$, that is, $T y=y$. Then

$$
a d(y, T y)+b d(z, T y)=b d(z, y) \leq d(z, y),
$$

so, by hypothesis, $d(y, z)=d(T y, T z) \leq r d(y, z)$. Hence $d(y, z)=0$. This is a contradiction.

Remark 1 For $r \in[0,1 / 2)$, taking $a=1, b=0$, we obtain Suzuki's condition from Theorem 2. Moreover, from our condition and the triangle inequality, we get

$$
a d(x, T x)+b[d(x, T x)-d(y, x)] \leq d(y, x),
$$

that is,

$$
\frac{a+b}{1+b} d(x, T x) \leq d(y, x)
$$

If $r \in[1 / \sqrt{2}, 1)$, we have

$$
\frac{a+b}{1+b}=\frac{1}{1+r}=\theta(r)
$$

hence our condition implies Suzuki's condition. We also note that if we take $a=(1-r) / r^{2}$, $b=0$ for $r \in[1 / 2,1 / \sqrt{2})$, we get Suzuki's condition. Therefore, our theorem generalizes, extends and complements Suzuki's theorem.

Example 1 Define a complete metric space $X$ by $X=\{-1,0,1,2\}$ and a mapping $T$ on $X$ by $T x=0$ if $x \in\{-1,0,1\}$ and $T 2=-1$. Then $T$ satisfies our condition from Theorem 7 for every $r \in[0,1 / 3) \cup[1 / 2,1)$, but $T$ does not satisfy Suzuki's condition from Theorem 2 .

Proof Since $\theta(r) d(1, T 1) \leq 1=d(1,2)$ for every $r \in[0,1)$, and $d(T 1, T 2)=1=d(1,2), T$ does not satisfy Suzuki's condition. If $r \in[1 / 2,(\sqrt{5}-1) / 2)$, we have $r^{2}+r<1$, so taking $a+b=$ $(1-r) / r^{2}$, we get $a+b>1$. Hence $a d(1, T 1)+b d(1, T 2)=a+2 b>1=d(1,2)$ and $a d(2, T 2)+$ $b d(2, T 1)=3 a+2 b>1=d(1,2)$. Now it is obvious that $T$ satisfies our condition. If $r \in$ $[(\sqrt{5}-1) / 2,1)$, we take $b=1 / 2$. We have two cases: $r \in[(\sqrt{5}-1) / 2,1 / \sqrt{2})$ and $r \in[1 / \sqrt{2}, 1)$. In the first case we put $a=\left(2-2 r-r^{2}\right) /\left(2 r^{2}\right)$ and in the second $a=(2-r) /(2+2 r)$. We have $a+2 b=1+a>1$ in both cases, so $T$ satisfies our condition. If $r \in[0,1 / 3)$ for $a=1$, $b=1 / 2$, it is obvious that $T$ satisfies our condition.

The following theorem is a generalization of Theorem 4 . 
Theorem 8 Let $(X, d)$ be a complete metric space, and let $T$ be a mapping on X. Assume that there exist $r \in[0,1), s>r$ such that

$$
\frac{s-r}{1+r} d(x, T x)+d(y, T x) \leq s d(y, x) \quad \text { implies } \quad d(T x, T y) \leq r d(x, y)
$$

for all $x, y \in X$. Then $T$ has a unique fixed point. Moreover, if $s \geq 1$, then $T$ has a unique fixed point.

Proof Let $u_{1} \in X$ and the sequence $u_{n}$ be defined by $u_{n+1}=T u_{n}$. Since

$$
0=d\left(u_{n+1}, T u_{n}\right) \leq s d\left(u_{n+1}, u_{n}\right)-\frac{s-r}{1+r} d\left(u_{n}, T u_{n}\right),
$$

we get from hypothesis $d\left(u_{n+1}, u_{n+2}\right) \leq r d\left(u_{n+1}, u_{n}\right)$ for all $n \geq 1$. Therefore, $d\left(u_{n+1}, u_{n+2}\right) \leq$ $r^{n} d\left(u_{1}, u_{2}\right)$ for all $n \geq 1$. Thus

$$
\sum_{n=1}^{\infty} d\left(u_{n+1}, u_{n}\right) \leq \sum_{n=1}^{\infty} r^{n-1} d\left(u_{1}, u_{2}\right)<\infty
$$

Hence $\left\{u_{n}\right\}$ is a Cauchy sequence. Since $X$ is complete, $\left\{u_{n}\right\}$ converges to some point $z \in X$.

Now, we will show that there exists a subsequence $\left\{u_{n(k)}\right\}$ of $\left\{u_{n}\right\}$ such that

$$
d\left(z, T u_{n(k)}\right) \leq s d\left(z, u_{n(k)}\right)-\frac{s-r}{1+r} d\left(u_{n(k)}, T u_{n(k)}\right)
$$

for all $k \geq 1$. Arguing by contradiction, we suppose that there exists a positive integer $v$ such that

$$
d\left(z, T u_{n}\right)>s d\left(z, u_{n}\right)-\frac{s-r}{1+r} d\left(u_{n}, T u_{n}\right)
$$

for all $n \geq v$. Then we have

$$
\begin{aligned}
d\left(z, u_{n+2}\right) & >s d\left(z, u_{n+1}\right)-\frac{s-r}{1+r} d\left(u_{n+1}, u_{n+2}\right) \\
& >s^{2} d\left(z, u_{n}\right)-s \cdot \frac{s-r}{1+r} d\left(u_{n}, u_{n+1}\right)-\frac{s-r}{1+r} d\left(u_{n+1}, u_{n+2}\right) \\
& \geq s^{2} d\left(z, u_{n}\right)-\frac{s-r}{1+r}\left[s d\left(u_{n}, u_{n+1}\right)+r d\left(u_{n}, u_{n+1}\right)\right] \\
& =s^{2} d\left(z, u_{n}\right)-\frac{s-r}{1+r}(s+r) d\left(u_{n}, u_{n+1}\right) .
\end{aligned}
$$

By induction, we get for all $n \geq v, p \geq 1$ that

$$
d\left(z, u_{n+p}\right)>s^{p} d\left(z, u_{n}\right)-\frac{s-r}{1+r}\left(s^{p-1}+s^{p-2} r+\cdots+r^{p-1}\right) d\left(u_{n}, u_{n+1}\right) .
$$

Then we have

$$
\begin{aligned}
d\left(z, u_{n+p}\right) & >s^{p} d\left(z, u_{n}\right)-\frac{s-r}{1+r} \cdot s^{p-1} \cdot \frac{1-(r / s)^{p}}{1-r / s} d\left(u_{n}, u_{n+1}\right) \\
& =s^{p}\left[d\left(z, u_{n}\right)-\frac{s-r}{1+r} \cdot \frac{1-(r / s)^{p}}{s-r} d\left(u_{n}, u_{n+1}\right)\right] .
\end{aligned}
$$


Hence

$$
s^{p}\left[d\left(z, u_{n}\right)-\frac{1-(r / s)^{p}}{1+r} d\left(u_{n}, u_{n+1}\right)\right]<d\left(z, u_{n+p}\right) .
$$

On the other hand,

$$
\begin{aligned}
d\left(u_{n+p}, u_{n}\right) & \leq d\left(u_{n}, u_{n+1}\right)+d\left(u_{n+1}, u_{n+2}\right)+\cdots+d\left(u_{n+p-1}, u_{n+p}\right) \\
& \leq\left(1+r+\cdots+r^{p-1}\right) d\left(u_{n}, u_{n+1}\right) \\
& =\frac{1-r^{p}}{1-r} d\left(u_{n}, u_{n+1}\right) .
\end{aligned}
$$

Letting $p \rightarrow \infty$, we get for all $n \geq 1$ that $d\left(z, u_{n}\right) \leq d\left(u_{n}, u_{n+1}\right) /(1-r)$. Thus

$$
d\left(z, u_{n+p}\right) \leq d\left(u_{n+p}, u_{n+p+1}\right) /(1-r) \leq r^{p} d\left(u_{n}, u_{n+1}\right) /(1-r) .
$$

By (6) and (7) we have for all $n \geq v, p \geq 1$ that

$$
\frac{r^{p}}{1-r} d\left(u_{n}, u_{n+1}\right)>s^{p}\left[d\left(z, u_{n}\right)-\frac{1-(r / s)^{p}}{1+r} d\left(u_{n}, u_{n+1}\right)\right],
$$

So

$$
\frac{(r / s)^{p}}{1-r} d\left(u_{n}, u_{n+1}\right)>d\left(z, u_{n}\right)-\frac{1-(r / s)^{p}}{1+r} d\left(u_{n}, u_{n+1}\right) .
$$

Taking the limit as $p \rightarrow \infty$, we obtain that $d\left(z, u_{n}\right) \leq d\left(u_{n}, u_{n+1}\right) /(1+r)$ for all $n \geq v$. Then we have

$$
d\left(z, u_{n+1}\right) \leq d\left(u_{n+1}, u_{n+2}\right) /(1+r) \leq r d\left(u_{n}, u_{n+1}\right) /(1+r)
$$

and

$$
r d\left(u_{n}, u_{n+1}\right) /(1+r)>s d\left(z, u_{n}\right)-(s-r) d\left(u_{n}, u_{n+1}\right) /(1+r) .
$$

This implies $d\left(z, u_{n}\right)<d\left(u_{n}, u_{n+1}\right) /(1+r)$ for all $n \geq v$. Thus,

$$
d\left(u_{n}, u_{n+1}\right) \leq d\left(z, u_{n}\right)+d\left(z, u_{n+1}\right)<d\left(u_{n}, u_{n+1}\right) /(1+r)+r d\left(u_{n}, u_{n+1}\right) /(1+r)=d\left(u_{n}, u_{n+1}\right) .
$$

This is a contradiction. Therefore there exists a subsequence $\left\{u_{n(k)}\right\}$ of $\left\{u_{n}\right\}$ such that

$$
d\left(z, T u_{n(k)}\right) \leq s d\left(z, u_{n(k)}\right)-\frac{s-r}{1+r} d\left(u_{n(k)}, T u_{n(k)}\right)
$$

for all $k \geq 1$. By hypothesis, we get $d\left(T z, T u_{n(k)}\right) \leq r d\left(z, u_{n(k)}\right)$. Letting $k \rightarrow \infty$, we obtain $d(T z, z)=0$, that is, $z=T z$.

If $s \geq 1$, we assume that $y$ is another fixed point of $T$. Then $d(z, T y)=d(z, y) \leq s d(z, y)-$ $(s-r) d(y, T y) /(1+r)=s d(z, y)$, so, by hypothesis, $d(z, y)=d(T z, T y) \leq r d(z, y)$. Since $r<1$, this is a contradiction. 


\section{Edelstein's theorem}

The following theorem extends Theorem 6 and generalizes Theorem 5 .

Theorem 9 Let $(X, d)$ be a compact metric space, and let $T$ be a mapping on $X$. Assume that

$$
a d(x, T x)+b d(y, T x)<d(y, x) \quad \text { implies } \quad d(T x, T y)<d(x, y)
$$

for $x, y \in X$, where $a>0, b>0,2 a+b<1$. Then $T$ has a unique fixed point.

Proof We put

$$
\beta=\inf \{d(x, T x): x \in X\}
$$

and choose a sequence $\left\{x_{n}\right\}$ in $X$ such that $\lim _{n \rightarrow \infty} d\left(x_{n}, T x_{n}\right)=\beta$. Since $X$ is compact, without loss of generality, we may assume that $\left\{x_{n}\right\}$ and $\left\{T x_{n}\right\}$ converge to some elements $v, w \in X$, respectively. We have

$$
\lim _{n \rightarrow \infty} d\left(x_{n}, w\right)=\lim _{n \rightarrow \infty} d\left(T x_{n}, v\right)=d(v, w)=\beta
$$

We shall show $\beta=0$. Arguing by contradiction, we assume $\beta>0$. Since

$$
\lim _{n \rightarrow \infty}\left[a d\left(x_{n}, T x_{n}\right)+b d\left(w, T x_{n}\right)\right]=a \beta<\beta=\lim _{n \rightarrow \infty} d\left(w, x_{n}\right),
$$

we can choose a positive integer $v$ such that

$$
a d\left(x_{n}, T x_{n}\right)+b d\left(w, T x_{n}\right)<d\left(w, x_{n}\right)
$$

for all $n \geq v$. By hypothesis, $d\left(T w, T x_{n}\right)<d\left(w, x_{n}\right)$ holds for $n \geq v$. This implies

$$
d(w, T w)=\lim _{n \rightarrow \infty} d\left(T w, T x_{n}\right) \leq \lim _{n \rightarrow \infty} d\left(w, x_{n}\right)=\beta
$$

From the definition of $\beta$, we obtain $d(w, T w)=\beta$. Since $a d(w, T w)+b d(T w, T w)<d(T w, w)$, we have

$$
d\left(T w, T^{2} w\right)<d(w, T w)=\beta
$$

which contradicts the definition of $\beta$. Therefore we obtain $\beta=0$. We have $\lim _{n \rightarrow \infty} d\left(x_{n}\right.$, $w)=\lim _{n \rightarrow \infty} d\left(T x_{n}, v\right)=\lim _{n \rightarrow \infty} d\left(T x_{n}, x_{n}\right)=d(v, w)=0$, so $v=w$. Thus, $\lim _{n \rightarrow \infty} x_{n}=$ $\lim _{n \rightarrow \infty} T x_{n}=w$.

We next show that $T$ has a fixed point. Arguing by contradiction, we assume that $T$ does not have a fixed point. Since $a d\left(x_{n}, T x_{n}\right)+b d\left(T x_{n}, T x_{n}\right)<d\left(T x_{n}, x_{n}\right)$ for all $n \geq$ 1, we get $d\left(T^{2} x_{n}, T x_{n}\right)<d\left(T x_{n}, x_{n}\right)$, so $\lim _{n \rightarrow \infty} T^{2} x_{n}=w$. By induction, we obtain that $d\left(T^{p} x_{n}, T^{p+1} x_{n}\right)<d\left(T^{p-1} x_{n}, T^{p} x_{n}\right)<\cdots<d\left(x_{n}, T x_{n}\right)$ and $\lim _{n \rightarrow \infty} T^{p} x_{n}=w$ for all integers $p \geq 1$. If there exist an integer $p \geq 1$ and a subsequence $\left\{x_{n(k)}\right\}$ of $\left\{x_{n}\right\}$ such that

$$
\operatorname{ad}\left(T^{p-1} x_{n(k)}, T^{p} x_{n(k)}\right)+b d\left(w, T^{p} x_{n(k)}\right)<d\left(w, T^{p-1} x_{n(k)}\right)
$$


for all $k \geq 1$, by hypothesis we get $d\left(T w, T^{p} x_{n(k)}\right)<d\left(w, T^{p-1} x_{n(k)}\right)$. Taking the limit as $k \rightarrow$ $\infty$, we obtain $d(w, T w)=0$, that is, $T w=w$, which is a contradiction. Hence, we can assume that for every $m \geq 1$, there exists an integer $n(m) \geq 1$ such that

$$
a d\left(T^{m-1} x_{n}, T^{m} x_{n}\right)+b d\left(w, T^{m} x_{n}\right) \geq d\left(w, T^{m-1} x_{n}\right)
$$

for all $n \geq n(m)$. Since

$$
\lim _{p \rightarrow \infty} \frac{p b^{p}}{1-b^{p}}=0
$$

and

$$
\frac{2 a}{1-b}<1
$$

we can choose $p$ satisfying

$$
\frac{p b^{p}}{1-b^{p}}+\frac{(p-1) b^{p-1}}{1-b^{p-1}}+\frac{2 a}{1-b}<1 .
$$

We put $v=\max \{n(1), n(2), \ldots, n(p)\}$. Then by (9) we have

$$
\begin{aligned}
d\left(w, x_{n}\right) \leq & a d\left(x_{n}, T x_{n}\right)+b d\left(w, T x_{n}\right) \\
\leq & a d\left(x_{n}, T x_{n}\right)+b\left[a d\left(T x_{n}, T^{2} x_{n}\right)+b d\left(w, T^{2} x_{n}\right)\right] \\
= & a d\left(x_{n}, T x_{n}\right)+a b d\left(T x_{n}, T^{2} x_{n}\right)+b^{2} d\left(w, T^{2} x_{n}\right) \\
\leq & \cdots \\
\leq & a d\left(x_{n}, T x_{n}\right)+a b d\left(T x_{n}, T^{2} x_{n}\right)+\cdots \\
& +a b^{p-1} d\left(T^{p-2} x_{n}, T^{p-1} x_{n}\right)+b^{p} d\left(w, T^{p} x_{n}\right) \\
\leq & \left(a+a b+\cdots+a b^{p-1}\right) d\left(x_{n}, T x_{n}\right)+b^{p} d\left(w, T^{p} x_{n}\right) \\
\leq & {\left[a\left(1-b^{p}\right) /(1-b)\right] d\left(x_{n}, T x_{n}\right)+b^{p} d\left(w, T^{p} x_{n}\right) }
\end{aligned}
$$

for all $n \geq v$. Since

$$
\begin{aligned}
d\left(w, T^{p} x_{n}\right) & \leq d\left(w, x_{n}\right)+d\left(x_{n}, T x_{n}\right)+\cdots+d\left(T^{p-1} x_{n}, T^{p} x_{n}\right) \\
& <d\left(w, x_{n}\right)+p d\left(x_{n}, T x_{n}\right),
\end{aligned}
$$

we get

$$
d\left(w, x_{n}\right)<\left[a\left(1-b^{p}\right) /(1-b)\right] d\left(x_{n}, T x_{n}\right)+b^{p}\left[d\left(w, x_{n}\right)+p d\left(x_{n}, T x_{n}\right)\right],
$$

so

$$
d\left(w, x_{n}\right)<\left(\frac{a}{1-b}+\frac{p b^{p}}{1-b^{p}}\right) d\left(x_{n}, T x_{n}\right)
$$


for all $n \geq v$. Similarly, we can obtain

$$
\begin{aligned}
d\left(w, T x_{n}\right) & <\left[\frac{a}{1-b}+\frac{(p-1) b^{p-1}}{1-b^{p-1}}\right] d\left(T x_{n}, T^{2} x_{n}\right) \\
& <\left[\frac{a}{1-b}+\frac{(p-1) b^{p-1}}{1-b^{p-1}}\right] d\left(x_{n}, T x_{n}\right)
\end{aligned}
$$

for all $n \geq v$. Using (11), we get

$$
d\left(x_{n}, T x_{n}\right) \leq d\left(w, x_{n}\right)+d\left(w, T x_{n}\right)<\left[\frac{2 a}{1-b}+\frac{p b^{p}}{1-b^{p}}+\frac{(p-1) b^{p-1}}{1-b^{p-1}}\right] d\left(x_{n}, T x_{n}\right)
$$

for all $n \geq v$. Thus, by (10), we obtain $d\left(x_{n}, T x_{n}\right)<d\left(x_{n}, T x_{n}\right)$, which is a contradiction. Therefore there exists $z \in X$ such that $T z=z$. Fix $y \in X$ with $y \neq x$. Then since $a d(x, T x)+$ $b d(y, T x)=b d(y, x)<d(y, x)$, we have $d(T y, x)=d(T y, T x)<d(y, x)$ and hence $y$ is not a fixed point of $T$. Therefore, the fixed point of $T$ is unique.

Remark 2 The proof of Theorem 9 is available for $a=1 / 2, b=0$. In this case we obtained Theorem 6 . We do not know if Theorem 9 is still correct for $a=0, b=1$, or, more generally, for $2 a+b=1$. This is an open question.

Example 2 Define a complete metric space $X$ by $X=\{A, B, C, D, E\}$ such that $d(A, B)=$ $d(A, C)=d(B, D)=d(C, D)=2, d(A, D)=d(B, C)=3, d(A, E)=d(C, E)=5 / 2, d(B, E)=$ $d(D, E)=1$ and a mapping $T$ on $X$ by $T A=B, T B=E, T C=D, T D=E, T E=E$. Then $T$ satisfies our condition from Theorem 9 for $a=1 / 8, b=2 / 3$, but $T$ does not satisfy Suzuki's condition from Theorem 6 .

Proof We have $d(A, C)=2=d(T A, T C)$ and $(1 / 2) d(A, T A)=1<d(A, C)=2$, so $T$ does not satisfy Suzuki's condition from Theorem 6. Moreover, we have $a d(A, T A)+b d(C, T A)=$ $a d(C, T C)+b d(A, T C)=2 a+3 b=9 / 4>d(A, C)$. It is now obvious that $T$ satisfies our condition from Theorem 9.

\section{Competing interests}

The author declares that they have no competing interests.

\section{Acknowledgements}

The author is highly indebted to the referees for their careful reading of the manuscript and valuable suggestions.

Received: 29 July 2013 Accepted: 28 October 2013 Published: 25 Nov 2013

\section{References}

1. Banach, S: Sur les opérationes dans les ensembles abstraits et leur application aux équation intégrales. Fundam. Math. 3, 133-181 (1922)

2. Caristi, J: Fixed point theorems for mappings satisfying inwardness conditions. Trans. Am. Math. Soc. 215, $241-251$ (1976)

3. Caristi, J, Kirk, WA: Geometric fixed point theory and inwardness conditions. Lect. Notes Math. 490, 74-83 (1975)

4. Ćirić, LB: A generalization of Banach's contraction principle. Proc. Am. Math. Soc. 45, 267-273 (1974)

5. Ćirić, LB: A new fixed-point theorem for contractive mappings. Publ. Inst. Math. (Belgr.) 30, 25-27 (1981)

6. Chauhan, S, Kadelburg, Z, Dalal, S: A common fixed point theorem in metric space under general contractive condition. J. Appl. Math. 2013, Article ID 510691 (2013)

7. Ekeland, I: On the variational principle. J. Math. Anal. Appl. 47, 324-353 (1974)

8. Imdad, M, Chauhan, S: Employing common limit range property to prove unified metrical common fixed point theorems. Int. J. Anal. 2013, Article ID 763261 (2013) 
9. Imdad, M, Chauhan, S, Kadelburg, Z: Fixed point theorems for mappings with common limit range property satisfying generalized $(\psi ; \phi)$-weak contractive conditions. Math. Sci. 2013, 7-16 (2013)

10. Kannan, R: Some results on fixed points II. Am. Math. Mon. 76, 405-408 (1969)

11. Kirk, WA: Contractions mappings and extensions. In: Kirk, WA, Sims, B (eds.) Handbook of Metric Fixed Point Theory, pp. 1-34. Kluwer Academic, Dordrecht (2001)

12. Kirk, WA: Fixed point of asymptotic contractions. J. Math. Anal. Appl. 277, 645-650 (2003)

13. Meir, A, Keeler, E: A theorem on contraction mappings. J. Math. Anal. Appl. 28, 326-329 (1969)

14. Nadler, SB Jr.: Multi-valued contraction mappings. Pac. J. Math. 30, 475-488 (1969)

15. Reich, S: Kannan's fixed point theorem. Boll. Unione Mat. Ital. 4, 1-11 (1971)

16. Subrahmanyam, PV: Remarks on some fixed point theorems related to Banach's contraction principle. J. Math. Phys. Sci. 8, 445-457 (1974)

17. Sehgal, VM, Bharucha-Reid, AT: Fixed points of contraction mappings on probabilistic metric spaces. Math. Syst. Theory 6, 97-102 (1972)

18. Khan, MA, Sumitra, Kumar, R Subcompatible and subsequential continuous maps in non Archimedean Menger PM-spaces. Jordan J. Math. Stat. 5, 137-150 (2012)

19. Khan, MA, Sumitra, Kumar, R: Semi-compatible maps and common fixed point theorems in non-Archimedean Menger PM-spaces. Jordan J. Math. Stat. 5, 185-199 (2012)

20. Suzuki, T: A generalized Banach contraction principle that characterizes metric completeness. Proc. Am. Math. Soc. $136,1861-1869(2008)$

21. Altun, I, Erduran, A: A Suzuki type fixed-point theorem. Int. J. Math. Math. Sci. 2011, Article ID 736063 (2011)

22. Karapinar, E, Tas, K: Generalized (C)-conditions and related fixed point theorems. Comput. Math. Appl. 61(11), 3370-3380 (2011)

23. Karapinar, E: Remarks on Suzuki (C)-condition. In: Dynamical Systems and Methods, pp. 227-243 (2012)

24. Kikkawa, M, Suzuki, T: Some similarity between contractions and Kannan mappings. Fixed Point Theory Appl. 2009, Article ID 192872 (2009)

25. Kikkawa, M, Suzuki, T: Three fixed point theorems for generalized contractions with constants in complete metric spaces. Nonlinear Anal. TMA 69, 2942-2949 (2008)

26. Moţ, G, Petruşel, A: Fixed point theory for a new type of contractive multivalued operators. Nonlinear Anal. TMA 70, 3371-3377 (2009)

27. Dhompongsa, S, Yingtaweesittikul, H: Fixed points for multivalued mappings and the metric completeness. Fixed Point Theory Appl. 2009, Article ID 972395 (2009)

28. Popescu, O: Two fixed point theorems for generalized contractions with constants in complete metric spaces. Cent. Eur. J. Math. 7, 529-538 (2009)

29. Popescu, O: A new type of multivalued contractive operators. Bull. Sci. Math. 137, 30-44 (2013)

30. Singh, SL, Mishra, SN: Remarks on recent fixed point theorems. Fixed Point Theory Appl. 2010, Article ID 452905 (2010)

31. Singh, SL, Pathak, HK, Mishra, SN: On a Suzuki type general fixed point theorem with applications. Fixed Point Theory Appl. 2010, Article ID 234717 (2010)

32. Singh, SL, Mishra, SN, Chugh, R, Kamal, R: General common fixed point theorems and applications. Fixed Point Theory Appl. 2012, Article ID 902312 (2012)

33. Edelstein, M: On fixed and periodic points under contractive mappings. J. Lond. Math. Soc. 37, 74-79 (1962)

34. Suzuki, T: A new type of fixed point theorem in metric spaces. Nonlinear Anal. 71, 5313-5317 (2009)

35. Dorić, D, Lazović, R: Some Suzuki type fixed point theorem for generalized multivalued mappings and applications. Fixed Point Theory Appl. 2011, Article ID 40 (2011)

36. Dorić, D, Kadelburg, Z, Radenović, S: Edelstein-Suzuki-type fixed point results in metric and abstract metric spaces. Nonlinear Anal. TMA 75(4), 1927-1932 (2012)

37. Karapinar, E: Edelstein type fixed point theorems. Fixed Point Theory Appl. 2012, Article ID 107 (2012)

38. Karapinar, E, Salimi, P: Suzuki-Edelstein type contractions via auxiliary functions. Math. Probl. Eng. 2013, Article ID $648528(2013)$

10.1186/1687-1812-2013-319

Cite this article as: Popescu: Some generalizations of Suzuki and Edelstein type theorems. Fixed Point Theory and Applications 2013, 2013:319

\section{Submit your manuscript to a SpringerOpen ${ }^{\ominus}$ journal and benefit from:}

- Convenient online submission

- Rigorous peer review

- Immediate publication on acceptance

Open access: articles freely available online

- High visibility within the field

- Retaining the copyright to your article

Submit your next manuscript at $>$ springeropen.com 